\title{
Design of the Nozzle for Producing Vortex Core-Spun Yarns
}

\author{
Zeguang Pei ${ }^{1 *}$, Cheng Jiang ${ }^{1}$, Changhe $\mathrm{Li}^{2}$ and Heju Ji ${ }^{3}$ \\ ${ }^{1}$ Donghua University, China \\ ${ }^{2}$ Qingdao University of Technology, China \\ ${ }^{3}$ Qingdao Dongjia Textile Machinery Group Co., Ltd, China
}

*Corresponding author: Zeguang Pei, Donghua University, Shanghai, China.

Received Date: October 09, 2020

Published Date: October 15, 2020

\begin{abstract}
The vortex spinning technology inserts twist into the yarn at a high speed by means of the swirling airflow generated inside the nozzle. The layered structure of its resultant yarn makes this spinning technology suitable for producing core-spun yarns. Therefore, the nozzle is the key part of the vortex spinning machine. In this paper, the current research progress of the design of the nozzle for producing vortex core-spun yarns is reviewed. The complex characteristics of the swirling airflow inside the nozzle are discussed. It is pointed out that numerical simulation based on computational fluid dynamics (CFD) is an effective method for optimizing the flow characteristics inside the nozzle and thus can provide the basis for designing the nozzle. Novel designs of the nozzle for producing vortex core-spun yarns are still expected, especially the nozzle with a low amount of lost fibers.
\end{abstract}

Keywords: Vortex spinning; Nozzle; Core-spun yarn; Airflow; Simulation

\section{Introduction}

Core-spun yarn is a type of composite yarn which consists of a continuous filament in the core and a sheath of twisted staple fibers wrapping around the filament. By combining the filament and staple fibers with different characteristics at the yarn level, the core-spun yarn can make full use of their advantages so that the resultant fabric can have the performance and style that cannot be realized by a single component [1]. In recent years, with the emergence of various functional fibers, core-spun yarns have provided a new way for the functionalization of fabrics for garments and have a broad application prospect in the fields of medical treatment, rehabilitation, national defense and energy, etc. For example, Wang et al. reported a core-spun yarn with an elastic polyurethane filament as the core and cotton fibers coated with carbon nanotubes as the sheath. The yarn works as a flexible strain sensor for sensing and recognizing human gestures so that it can be used for monitoring the prognosis of patients with limb injury and the daily movement of the elderly [2]. Ahmad et al. reported a core-spun yarn with an NiTi SMA wire as the core and cotton fibers as the sheath [3]. This type of yarn can be made into a planar spiral shape and fixed in the interlayer of the fire suit. The shape memory characteristic of the yarn can make it return to the shape of a spring under higher temperature, thus forming a large gap between two layers of fabrics to isolate the high temperature to prevent firefighters from scalding. Duran et al. reported a corespun yarn with silver plated nylon filament as the core and cotton fibers as the sheath. The fabrics made from the yarns can be used in electromagnetic shielding military suit and radiation-proof maternity clothing [4].

As stated above, with the expansion of the application fields of core-spun yarns, the highly reliable large-scale mass production methods for the core-spun yarns are in urgent demand. The methods mainly include ring spinning, rotor spinning, friction spinning and 
vortex spinning [3-5]. Among them, the vortex spinning technology adopts high-speed swirling airflow generated in a nozzle to insert twist into the fiber strand to form the yarn. This spinning technology features the highest production rate (up to $500 \mathrm{~m} / \mathrm{min}$ ) of all spinning methods and the yarn with a layered structure consisting of twist less fibers in the core wrapped by helical wrapper fibers. The unique yarn structure makes vortex spinning technology an excellent candidate for producing core-spun yarns in which the core filament can be well covered by the wrapper fibers in the outer layer [6]. Nozzle is the key part in the vortex spinning machine and the design of its structure has a significant effect on the resultant yarn properties and the performance of the machine. Therefore, this paper reviews the current progress of the design of the nozzle for producing the vortex core-spun yarns.

\section{Current Progress of the Design of the Nozzle}

The twist insertion into the fiber strand in vortex spinning is closely related to the swirling airflow characteristics inside the nozzle. Therefore, investigation into the flow characteristics is the important premise for the design of the nozzle. In the nozzle, the compressed air expands and is continuously accelerated inside several tangential injectors arranged along the circumference of the nozzle chamber. The air is then injected into the chamber at a velocity higher than the speed of sound (Ma>1). Due to the relay, entrainment and mixing of the air jets, the air in the vicinity and downstream of the injectors starts to move and then whirls around the axis of the chamber. According to the research result of Sun et al. [7], the swirling flow inside the nozzle exhibits a typical characteristic of Rankine vortex, i.e., the tangential velocity is composed of the forced vortex near the nozzle axis and the free vortex near the nozzle wall, while for a compressible flow, the area occupied by the free vortex is very small. The generated swirling flow is three-dimensional and strongly anisotropic [8]. In the radial direction, the formed shear layer has a very large velocity gradient. According to the numerical results provided by Guo et al. [9], the swirling flow gradually decays as it moves downstream along the axial direction, while an oscillation of its velocity along the axial direction can be observed. This results from the interaction and mixing between the air jets and the swirling flow. Chang F, et al. [10] found through experiment that there is a positive pressure gradient in the center of the strong swirling flow. It can lead to the change of the direction of the axial velocity in the central region so that a reverse flow can be generated, while an obvious shear layer exists at the boundary of the reverse flow. In the nozzle of vortex spinning, the reverse flow can be more obvious owing to the hinderance caused by the spindle. In the region with strong reverse flow, vortex breakdown can occur [11,12]. Vortex breakdown is one of the main characteristics of swirling flow. It is characterized as the axial reverse flow and rapid expansion of the vortex core, accompanied by the fluctuation of the location of the breakdown and the flow field in the downstream. Brücker C [13] found that vortex breakdown is similar to a bubble in shape, while its inner structure has a spiral characteristic. It then looks like a wake after it breaks down. The phenomenon of vortex breakdown is very complex. There is mass exchange between the flows inside and outside it, while it is unsteady, periodic and quite unstable to external disturbance. According to Pei Z, et al. [14], the Reynolds number of the airflow in the nozzle of vortex spinning is in the order of $10^{5}$ so that the flow is in the turbulent regime. The turbulence can result in the fluctuation of the physical quantities of the flow filed. The flow characteristic then becomes more complex owing to the interaction among the shear layer, vortex breakdown and turbulence intensity - the intensification of the vortex breakdown can increase the velocity gradient of the shear layer and at the same time significantly increase the turbulence intensity [15]. Therefore, it can be found that the swirling airflow characteristics in the nozzle of vortex spinning are very complex.

In view of the important role played by the nozzle in the vortex spinning machine, some new designs of the nozzle have been proposed recently based on the flow characteristics inside it. For example, Yan et al. carried out numerical simulation on the airflow field in the nozzle of vortex spinning machine with spiral guiding grooves on the outer surface of the spindle to analyze the influence of the grooves on the flow characteristics [16]. Han C, et al. studied the effect of guiding needle of the fiber guiding member on the flow characteristics using computational fluid dynamics (CFD) [17]. Shang, et al. [18] and Phung TVB, et al. [19] carried out three-dimensional numerical simulation and theoretical analysis on the flow field in the nozzle at machine startup, respectively. The studies above show that flow analysis techniques based on computational fluid dynamics are playing important roles in studying the flow characteristics in the nozzle of vortex spinning. However, the attention of current studies has been mainly paid to the time-averaged flow characteristics, while the transient flow characteristics are yet to be revealed. In the meantime, experimental studies on the flow characteristics are still scarce [7]. Therefore, study on the unsteady flow characteristics in the nozzle of vortex spinning still needs to be carried out. As to the nozzle for producing vortex core-spun yarns, in the traditional design, the filament enters the nozzle chamber together with the staple fibers through the spiral entrance at the nozzle inlet, which results in a long conveying path for the filament in the nozzle. Meanwhile, extra bending and twisting deformations can be expected for the filament as it moves around the guiding needle, which results in the difficulty of accurately controlling the tension of the filament as well as its position in the staple fiber strand. Therefore, a filament feeding device with an extremely complex structure is needed for controlling the tension [20]. Pei Z, et al. reported a design of the nozzle for producing vortex core-spun yarns [5,21]. In their design, a filament feeding orifice is provided through the fiber guiding member at the nozzle inlet instead of the guiding needle. The filament enters the nozzle chamber in a straight path along the nozzle axis, which then shortens the conveying path of the 
filament inside the nozzle and eliminates the extra bending and twisting deformations of the filament. However, the yarn formation mechanism in this design still needs to be further revealed in order to obtain yarns with stable structure and quality. Besides, in the nozzle of traditional design, some fibers are pulled out of the fiber strand under the action of the tangential and axial components of the forces from the swirling airflow inside the nozzle chamber and the annular cavity between the spindle and nozzle wall. These fibers become the lost fibers the two ends of which are both in the free state and are then expelled out of the nozzle, which can lead to the deterioration of the yarn evenness $[21,22]$. Therefore, in order to obtain vortex core-spun yarns with uniform staple fiber sheath around the core filament, designing the nozzle with a low amount of lost fibers is needed.

\section{Conclusions}

This paper reviews the current progress of the design of nozzle for producing vortex core-spun yarns. In summary, numerical simulations based on computational fluid dynamics (CFD) can be carried out to investigate the high-speed, compressible, unsteady and turbulent flow field inside the nozzle for the theoretical analysis of the flow characteristics. Experimental studies are also needed for the verification of the numerical results to make the flow characteristics inside the nozzle more reasonable. Based on these studies, nozzles with a low amount of lost fibers for producing vortex core-spun yarns can be developed. This will be our future work.

\section{Acknowledgement}

None.

\section{Conflict of Interest}

Authors declare no conflict of interest.

\section{References}

1. Varghese N, Thilagavathi G (2016) Handle, fit and pressure comfort of silk/hybrid yarn woven stretch fabrics. Fibers and Polymers 17(3): 484494.

2. Wang Z, Huang Y, Sun J, Huang Y, Hu H, et al. (2016) Polyurethane/ cotton/carbon nanotubes core-spun yarn as high reliability stretchable strain sensor for human motion detection. ACS Applied Materials \& Interfaces 8: 24837-24843.

3. Ahmad MR, Yahya MHM, Hassan MR, Salleh J, Ahmad WYW, et al. (2013) Production of shape memory alloy core-sheath friction yarns. Fibres \& Textiles in Eastern Europe 21(3): 68-72.

4. Duran D, Kadoğlu H (2015) Electromagnetic shielding characterization of conductive woven fabrics produced with silver-containing yarns. Textile Research Journal 85(10): 1009-1021.

5. Pei Z, Zhang Y, Chen G (2019) A core-spun yarn containing a metal wire manufactured by a modified vortex spinning system. Textile Research Journal 89(1): 113-118.
6. Ortlek HG, Ulku S (2007) Effects of spandex and yarn counts on the properties of elastic core-spun yarns produced on Murata vortex spinner. Textile Research Journal 77(6): 432-436.

7. Sun L, Pei Z (2016) Effects of structural parameters on the tangentially injected swirling flow in concentric tubes with different lengths as a model of the vortex spinning nozzle. Textile Research Journal 86(12): 1241-1258.

8. Guo HF, Chen ZY, Yu CW (2009) 3D numerical simulation of compressible swirling flow induced by means of tangential inlets. International Journal for Numerical Methods in Fluids 59: 1285-1298.

9. Guo HF, Yu CW, Xu BG, Li SY (2011) Effect of the geometric parameters on a flexible fiber motion in a tangentially injected divergent swirling tube flow. International Journal of Engineering Science 49(10): 10331046 .

10. Chang F, Dhir VK (1995) Mechanisms of heat transfer enhancement and slow decay of swirl in tubes using tangential injection. International Journal of Heat and Fluid Flow 16(2): 78-87.

11. Lucca-Negro O, O’Doherty T (2001) Vortex breakdown: a review. Progress in Energy and Combustion Science 27(4): 431-481.

12. Guo HF, Chen ZY (2009) Numerical simulation of tangentially injected turbulent swirling flow in a divergent tube. International Journal for Numerical Methods in Fluids 61(7): 796-809.

13. Brücker C (2002) Some observations of vortex breakdown in a confined flow with solid body rotation. Flow Turbulence and Combustion 69(1): 63-78.

14. Pei Z, Yu C (2009) Study on the principle of yarn formation of Murata vortex spinning using numerical simulation. Textile Research Journal 79(14): 1274-1280

15. Guo HF, Xu BG, Yu CW, Li SY (2011) Simulating the motion of a flexible fiber in 3D tangentially injected swirling airflow in a straight pipe effects of some parameters. International Journal of Heat and Mass Transfer 54(21-22): 4570-4579.

16. Yan L, Zou Z, Cheng L, Wei G, Tang P (2019) Numerical simulation of flow field in the twisting chamber of Murata vortex spinning based on the hollow spindle with different structures. Textile Research Journal 89(4): 645-656.

17. Han C, Cheng L, Gao W, Xue Y, Xue W, etal. (2019) Analysis of the influence of the guided needle structure on the vortex spinning process and yarn properties. Textile Research Journal 89(7): 1246-1257.

18. Shang S, Yang J, Yu C (2019) Numerical simulation of the airflow field in vortex spinning process. Textile Research Journal 89(6): 1113-1127.

19. Phung TVB, Yoshida A, Iemoto Y, Uematsu H, Tanoue S (2019) Numerical simulation of airflow characteristics in the spinning zone at starting time of air-jet spinning machine. Textile Research Journal 89(12): 23422352.

20. Sawada H, Akimoto M (2017) Core yarn supplying device, spinning machine, and method of supplying core yarn. European patent: EP 3040458 B1.

21. Pei Z, He J (2019) Experimental study on the formation of core-spun yarn manufactured on a modified vortex spinning system. Textile Research Journal 89(21-22): 4383-4397.

22. Erdumlu N, Ozipek B, Oxenham W (2012) Vortex spinning technology. Textile Progress 44(3-4): 141-174. 